\title{
Neue teilretardierte Formulierung bei Rosacea
}

— Rosacea ist eine der häufigsten Hauterkrankungen im Erwachsenenalter.,,Leichte bis moderate Formen der Rosacea können mit topischen Therapeutika, mittlere bis schwere Formen sollten mit systemischen Wirkstoffen, wie zum Beispiel Doxycyclin, behandelt werden", sagte Prof. Dr. Dieter Reinel aus Hamburg
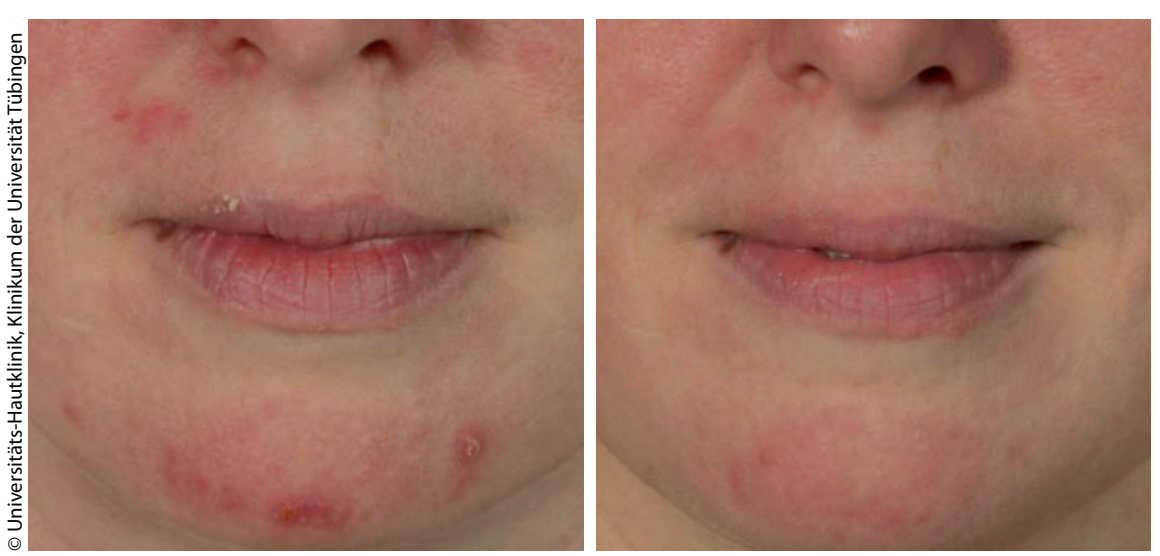

Patientin vor der Behandlung (links) und nach vier Wochen (rechts) mit teilretardiertem,

niedrig dosiertem Doxycyclin und topisch angewandtem Metronidazol auf der 13. Dermatologischen Wissenschaftsund Fortbildungsakademie in Köln. Eine langfristige Therapie der Rosacea, zum Beispiel mit 100 mg Doxycyclin über mehrere Wochen, birgt jedoch einige Risiken:„Es kann zu Superinfektionen, wie vaginalen Candidosen, Lichtempfindlichkeit, gastrointestinalen Neben- wirkungen und einer bakteriellen Arzneimittelresistenz kommen", beschrieb Reinel. Nicht so bei der neuen teilretardierten Formulierung des Doxycyclin, die nur antientzündlich, nicht aber antimikrobiell wirkt: Oraycea ${ }^{\circledR}$ enthält $40 \mathrm{mg}$ Doxycyclin, wobei $30 \mathrm{mg}$ schnell und $10 \mathrm{mg}$ verzögert freigesetzt werden. So kommt es zu einem konstant subantimikrobiellen Plasmaspiegel über 24 Stunden. Dessen Wirksamkeit bei der Behandlung von RosaceaPatienten ist vergleichbar mit der von $100 \mathrm{mg}$ Doxycyclin (Abb.). „Aber die Verträglichkeit ist deutlich besser", erklärte Reinel. So kam es in Studien unter $40 \mathrm{mg}$ Oraycea ${ }^{\circledR}$ deutlich seltener zu gastrointinalen Beschwerden als bei $100 \mathrm{mg}$ Doxycyclin. Auch wurden mit Oraycea ${ }^{\circledast}$ bislang keine Ausbildung von Resistenzen, keine Candida-Infektionen oder Lichtempfindlichkeiten beobachtet. Es ist zur Behandlung von papulopustulöser Rosacea bei Erwachsenen zugelassen. Dr. Caroline Steingen

Satellitensymposium „Antiinflammatorisch gegen Rosacea" anlässlich der 13. Dermatologischen Wissenschafts- und Fortbildungsakademie, Köln, 27. November 2010 (Veranstalter: Galderma)

\section{Neuartige Wundauflage fördert die Heilung}

_ Die Behandlung chronischer Wunden lässt sich durch eine innovative Wundauflage fördern. Die neuartige Wundversorgung (UrgoCell ${ }^{\circledR}$ Contact) basiert auf der sogenannten Lipidokolloid-Technologie (TLC), bei der Hydrokolloidpartikel und Vaseline in ein Netz aus Polyesterfasern eingebracht werden. Die Hydrokolloidpartikel verflüssigen sich im Kontakt mit dem Wundsekret, sodass ein Gel entsteht, das mit der Vaseline einen Lipidkolloidfilm an der Grenzschicht zwischen Wunde und Wundauflage bildet. Damit lässt sich ein gleichbleibend feuchtes Wundmilieu erwirken. Die neue Technologie sorgt zugleich dafür, dass der Verbandswechsel atraumatisch und damit deutlich weniger schmerzhaft erfolgt als bisher.

Die TLC-Technologie hat aber noch weitere Vorteile, die PD Dr. Axel Larena-Avellaneda, Hamburg, bei einer Pressekonferenz in Essen darlegte. Sie erlaubt zum Beispiel die Einlagerung antibakteriell wirkender Silberkomponenten, wodurch sich Wundinfektionen entgegenwirken lässt.
Eine weitere innovative Wundauflage (UrgoCell ${ }^{\circledR}$ START) enthält den Proteaseinhibitor NOSF (Nano-Oligosaccharidfaktor), der die Aktivität überschüssiger Proteasen hemmt. Er verhindert so, dass gesundes Gewebe sowie für die Wundheilung essenzielle Wachstumsfaktoren abgebaut werden. Das ist laut LarenaAvellaneda vor allem bei der Behandlung chronischer Wunden hilfreich: „Durch den Einsatz dieses Wundmilieu-modulierenden Faktors wird die in solchen Wunden gestörte Wundheilung günstig beeinflusst und die Granulation wieder initiiert", erklärte LarenaAvellaneda in Essen.

Christine Vetter

Pressekonferenz „10 Jahre Lipidokolloid-Technologie (TLC) in der hydroaktiven Wundversorgung: Rückblick und Ausblick". Essen, 29. September 2010 (Veranstalter: Urgo Medical)

\section{Hautpflege für den Frühling}

— Vom Winter beanspruchte Haut wird jetzt mit einem intensiven Pflegeprogramm verwöhnt. Die Eucerin ${ }^{\circledast}$ Hautglättende Lotion 3\% Urea enthält Feuchtigkeit spendende und bindende Inhaltsstoffe wie Urea, Glycerin und Lactat sowie Carnitin, die helfen, die trockene Haut wieder in Balance zu bringen. Die Lotion ist besonders hautverträglich und eignet sich für alle Formen trocke- ner Haut - auch bei Neurodermitis im erscheinungsfreien Intervall und bei Psoriasis. Sie enhält keine Duftstoffe.

Als einzige von 17 Körperlotions wurde Eucerin ${ }^{\oplus}$ Hautglättende Lotion 3\% Urea von Stiftung Warentest mit der Bestnote "sehr gut" ausgezeichnet (test 9/2010).

Nach Informationen von Beiersdorf 